\title{
Apresentação do Grupo de Pesquisas em Ciências Cognitivas e Tecnologias Educacionais
}

\author{
Alex Sandro Gomes', Ricardo José Rocha Amorim², Rodrigo Lins Rodrigues ${ }^{3}$, \\ Jorge Luis Cavalcanti Ramos ${ }^{4}$ \\ ${ }^{1}$ Centro de Informática - Universidade Federal de Pernambuco - UFPE \\ ${ }^{2}$ Faculdade de Ciências Aplicadas e Sociais de Petrolina - FACAPE \\ ${ }^{3}$ Departamento de Educação - Universidade Federal Rural de Pernambuco - UFRPE \\ ${ }^{4}$ Colegiado de Engenharia de Computação - Universidade Federal do Vale do São Francisco-UNIVASF \\ asg@cin.ufpe.br, amorim.ricardo@gmail.com, \\ jorge.cavalcanti@univasf.edu.br, rlr@ded.ufrpe.br
}

\section{Identificação do Grupo}

Nome: Ciências Cognitivas e Tecnologia Educacional (CCTE)

Diretório CNPQ: dgp.cnpq.br/dgp/espelhogrupo/6795353807774630

Website: www.cin.ufpe.br/ ccte

\section{Descrição do Grupo}

Objetivos: As pesquisas realizadas pelo grupo envolvem temas relativos a análise e ao desenvolvimento de tecnologias educacionais a serem utilizadas na formação de recursos humanos em diversas áreas do conhecimento. A elaboração de nossas soluções tecnológicas é sempre fundamentada pela criação de modelos teóricos de ação, atividade, aprendizagem e interação que ajudam a modelar e que permitem a correta implementação de sistemas computacionais.

Da mesma forma, nossa tradição metodológica considera as características cognitivas dos usuários e do contexto de uso das tecnologias ao ponto de envolvê-los no processo do projeto de forma iterativa, visando a melhoria da usabilidade dos sistemas criados.

Áreas de atuação: Ciências Cognitivas e Tecnologia Educacional, Educação a Distância, Groupware, Inteligência Artificial e Educação, Interfaces Educativas e Interfaces Inteligentes.

\section{Instituições Participantes}

Universidade Federal de Pernambuco, Universidade Federal Rural de Pernambuco, Universidade Federal do Vale do São Francisco, Universidade Federal Rural do Semiárido, Faculdade de Ciências Aplicadas e Sociais de Petrolina, Universidade do Estado da Bahia, Instituto Federal de Educação, Ciência e Tecnologia de Pernambuco, Instituto Federal de Educação, Ciência e Tecnologia do Sertão Pernambucano, Instituto Federal de Educação, Ciência e Tecnologia da Paraíba.

\section{Pesquisadores envolvidos}

Líderes do Grupo: Alex Sandro Gomes (CIn-UFPE) e Ricardo Amorim (FACAPE)

Pesquisadores: 13 Estudantes pós-graduação: 15 Estudantes graduação: 09

\section{Projetos desenvolvidos e resultados alcançados}

Ambientes Virtuais de Aprendizagem: Amadeus LMS, Amadeus Droid, Amadeus Droid Dislexia, Redu, Redu Mobile.

Produção Científica: 16 artigos em periódicos, 96 artigos em anais de eventos, 02 livros, 04 capítulos de livros. 